\title{
Improved coherence of ultrafast spin- transfer-driven precessional switching with synthetic antiferromagnet perpendicular polarizer
}

Cite as: Appl. Phys. Lett. 98, 242511 (2011); https://doi.org/10.1063/1.3597797

Submitted: 14 March 2011 . Accepted: 17 May 2011 . Published Online: 17 June 2011

A. Vaysset, C. Papusoi, L. D. Buda-Prejbeanu, S. Bandiera, M. Marins de Castro, Y. Dahmane, J.-C. Toussaint, U. Ebels, S. Auffret, R. Sousa, L. Vila, and B. Dieny

\section{ARTICLES YOU MAY BE INTERESTED IN}

Precessional spin-transfer switching in a magnetic tunnel junction with a synthetic antiferromagnetic perpendicular polarizer

Journal of Applied Physics 111, 07 C912 (2012); https://doi.org/10.1063/1.3676610

100 ps precessional spin-transfer switching of a planar magnetic random access memory cell with perpendicular spin polarizer

Applied Physics Letters 95, 072506 (2009); https://doi.org/10.1063/1.3206919

Spin torque switching of perpendicular $\mathrm{Ta}|\mathrm{CoFeB}| \mathrm{MgO}$-based magnetic tunnel junctions Applied Physics Letters 98, 022501 (2011); https://doi.org/10.1063/1.3536482
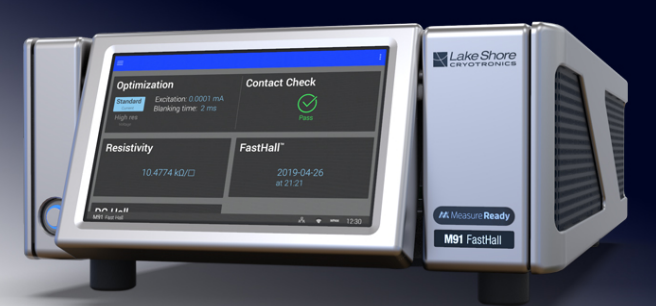

\section{Measure Ready M91 FastHall ${ }^{\text {TM }}$ Controller}

A revolutionary new instrument for complete Hall analysis See the video 0

LakeShore 


\title{
Improved coherence of ultrafast spin-transfer-driven precessional switching with synthetic antiferromagnet perpendicular polarizer
}

\author{
A. Vaysset, ${ }^{1, a)}$ C. Papusoi, ${ }^{1}$ L. D. Buda-Prejbeanu, ${ }^{1,2}$ S. Bandiera, ${ }^{1}$ M. Marins de Castro, ${ }^{1}$ \\ Y. Dahmane, ${ }^{1}$ J.-C. Toussaint, ${ }^{2,3}$ U. Ebels, ${ }^{1}$ S. Auffret, ${ }^{1}$ R. Sousa, ${ }^{1}$ L. Vila, ${ }^{4}$ and \\ B. Dieny ${ }^{1}$ \\ ${ }^{1}$ SPINTEC, INAC, UMR CEA/CNRS/UJF-Grenoble 1/Grenoble-INP, Grenoble F-38054, France \\ ${ }^{2}$ Grenoble INP, 46, Avenue Félix Viallet, 38031 Grenoble Cedex 1, France \\ ${ }^{3}$ Institut Néel, CNRS and Université Joseph Fourier, F-38042 Grenoble, France \\ ${ }^{4}$ SP2M/NM, INAC, CEA-Grenoble, 38054 Grenoble Cedex, France
}

(Received 14 March 2011; accepted 17 May 2011; published online 17 June 2011)

\begin{abstract}
The coherence of the precessional switching was compared in planar spin-valves comprising either an additional simple perpendicular polarizer or a synthetic antiferromagnet perpendicular polarizer. A significant improvement in the precession coherence was observed experimentally in the second type of samples. Micromagnetic simulations were performed to study the effect of the stray field from the perpendicular polarizer. They provide an explanation for the gradual loss of coherence of the precession in terms of vortex formation, which occurs much faster when a simple perpendicular polarizer is used. (C) 2011 American Institute of Physics. [doi:10.1063/1.3597797]
\end{abstract}

The spin transfer torque (STT) was predicted by Slonczewski and Berger. ${ }^{1,2}$ When a spin-polarized current flows through a ferromagnetic layer, it exerts a torque on its magnetization. This effect can be used as a write scheme in memories or to generate steady magnetic excitations in frequency tunable RF nano-oscillators. ${ }^{3}$ STT-magnetic random access memory (STT-MRAM) is a very promising candidate for future memories thanks to its energy-efficiency, scalability, and high write speed. Switching has been studied in inplane magnetized structures ${ }^{4}$ by reversing the free layer (FL) magnetization with short current pulses (100 ps-10 ns). Stochastic variations in the switching time were observed ${ }^{5}$ in structures where the equilibrium directions of magnetization of the storage layer and of the reference layer lie along the same direction. Indeed the STT is zero as long as the magnetizations in the storage layer and the reference layer remain parallel or antiparallel. Therefore, the reversal is triggered when the angle of the FL magnetization is sufficiently large. This angle strongly depends on thermal fluctuations. Several solutions were proposed to decrease or suppress this so-called incubation delay: applying a dc current ${ }^{6}$ or a microwave-frequency pulse ${ }^{7}$ before a square current pulse, applying a small in-plane hard-axis field, ${ }^{8-10}$ or changing the equilibrium angle between the free and the pinned layer. ${ }^{11-13}$ Using a double-pulse was also proposed to achieve a more reliable switching on the subnanosecond time scale. ${ }^{14}$

Another strategy to avoid these stochastic effects consists in adding to the planar magnetic tunnel junction a perpendicular polarizer (PL) ${ }^{15-17}$ When the current is applied, the FL magnetization is pulled out-of-plane and precesses about the out-of-plane axis, due to the STT from the perpendicular polarizer. If the current is stopped, the magnetization returns rapidly $(<400 \mathrm{ps})$ into the plane and relaxes in one of its two in-plane equilibrium states. Depending on the pulse duration, it is thus possible to switch the magnetization or bring it back to its initial state. If the magnetization achieves a half precessional cycle, it switches in a very short

${ }^{a)}$ Electronic mail: adrien.vaysset@cea.fr. time of the order of 100-300 ps varying as the inverse of the current density amplitude. ${ }^{18-20}$ Moreover, the reversal starts as soon as the current is applied, without incubation delay. A 100 ps switching was thus experimentally demonstrated. ${ }^{20,21}$ It is a great step forward in the context of ultrafast memories or logic devices. Nevertheless, when using a single perpendicular polarizer, a rapid loss of coherence in the precessional motion was observed with an associated risk of irreproducibility in the magnetization switching. Indeed, when increasing the pulse duration to perform several precessional cycles, a very fast decay in the amplitude of oscillations of the switching probability was observed. ${ }^{21}$ This observation could be ascribed to the role of thermal fluctuations, Oersted field, or stray field from the perpendicular polarizer.

In this letter, we report on experiments which show a strong improvement in the coherence of the precessional motion when the stray field from the perpendicular polarizer is reduced by using a synthetic antiferromagnet (SAF) for the perpendicular polarizer. We compare these results to micromagnetic simulations in order to understand the effect of the stray field on the FL magnetization dynamics.

The structure of the investigated samples was $\mathrm{PL}_{\perp} / \mathrm{Cu} 4 / \mathrm{FL} / \mathrm{Cu} 4 / \mathrm{AL}$, where the perpendicular polarizer $\mathrm{PL}_{\perp}$ is $\mathrm{Pt} 15 /(\mathrm{Co} 0.5 / \mathrm{Pt} 0.4)_{\times 7} / \mathrm{Co} 1 / \mathrm{Ru} 0.9 /$ (Co $0.5 / \mathrm{Pt} 0.4)_{\times 5} / \mathrm{Co} 1 / \mathrm{Cu} 0.3 / \mathrm{Co} 1$, the FL is Co $0.5 /$ $\mathrm{Ni}_{80} \mathrm{Fe}_{20} 1.5 / \mathrm{Co} 0.5$, and the analyzing layer (AL) is Co $0.5 / \mathrm{CoFeB}_{20} 1.5 / \mathrm{Ru} 0.9 / \mathrm{Co} 2 / \mathrm{IrMn} 7$ (all thicknesses are in nanometers). The PL and the $\mathrm{AL}$ are both compensated SAFs. The sample has an almost circular cross section of $100 \mathrm{~nm}$ diameter. Its current-perpendicular-to-plane magnetoresistance is $0.3 \%$ due to the large serial resistance of the buffer and capping layers. All measurements were performed at room temperature, with no applied magnetic field. The experimental setup is the same as the one described in Ref. 21.

The rise time of the current pulse was $50 \mathrm{ps}$ and the fall time was $100 \mathrm{ps}$. The static resistance was measured immediately after the end of the pulse. It was always very close to either $\mathrm{R}_{\mathrm{P}}$ or $\mathrm{R}_{\mathrm{AP}}$, indicating that, at the end of the switching, 


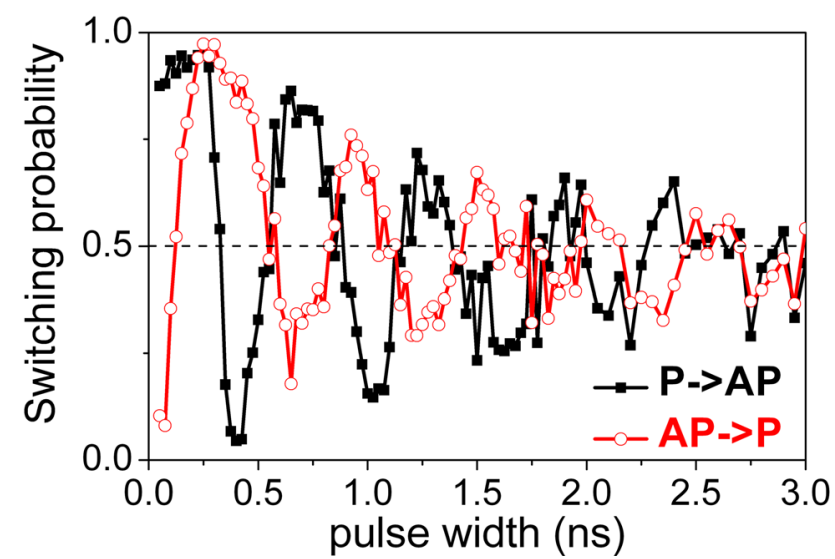

FIG. 1. (Color online) Switching probability as a function of pulse width. Squares: the initial state is parallel. Open circles: the initial state is antiparallel. The current density is $8.3 \times 10^{11} \mathrm{~A} / \mathrm{m}^{2}$.

the FL magnetization is uniform and aligned along the easy axis. The switching probability is plotted in Fig. 1 as a function of the pulse duration. Each point is averaged from 120 measurements. This plot must be compared with Fig. 3 in Ref. 21. With the SAF-PL, more than five oscillations in switching probability are observed before settling at 50\% probability corresponding to equal probability of ending up in the P or AP configurations. In contrast, when a single-layer perpendicular polarizer was used, barely two oscillation periods could be observed. Furthermore, the first "peak" almost reaches unity here, indicating an improved reproducibility in the switching.

The symmetry of the oscillations around $\mathrm{P}_{\mathrm{s}}=0.5$ indicates that the magnetization dynamics is dominated by the spin transfer from the perpendicular polarizer PL and that the in-plane pinned layer AL has a negligible influence on the switching dynamics likely due to a higher current polarization from the PL.

In order to understand the difference of coherence in the precessional motion between a single-layer PL and a SAF PL, micromagnetic simulations were performed. For simplicity the STT from the analyzer was neglected, which is consistent with the experimental observations. The dynamics was therefore modeled as being driven solely by the spin torque from the out-of-plane polarizer. Micromagnetic simulations were performed using Finite Difference method with our own software: ST_GL-FFT. ${ }^{22}$ In the simulations, the FL was an ellipse of dimensions $100 \times 90 \times 2.5 \mathrm{~nm}^{3}$, providing a slight in-plane uniaxial anisotropy such that the system has two single domain equilibrium states. The FL is discretized in a mesh with cells of $1.6 \times 1.4 \times 2.5 \mathrm{~nm}^{3}$. All the simulations were performed at zero temperature. Therefore, the system is totally deterministic: when a simulation is performed several times, the same result is always found. $\mathrm{P}_{\mathrm{s}}$ has then only two possible values: 0 and 1 .

Two systems were simulated: (i) the FL experiences only the STT from the polarizer (SAF-polarizer case); (ii) the FL experiences the STT and the stray field from the polarizer (single-layer polarizer case). ${ }^{21}$ No stray field from the analyzer was introduced since in Ref. 21 a compensation field was applied in the plane. Moreover, in the recent experiments, the analyzer is a compensated SAF. The stray field from the single layer polarizer was numerically calculated. It ranges from 60 to $150 \mathrm{kA} / \mathrm{m}$ in the FL, which is fairly large.
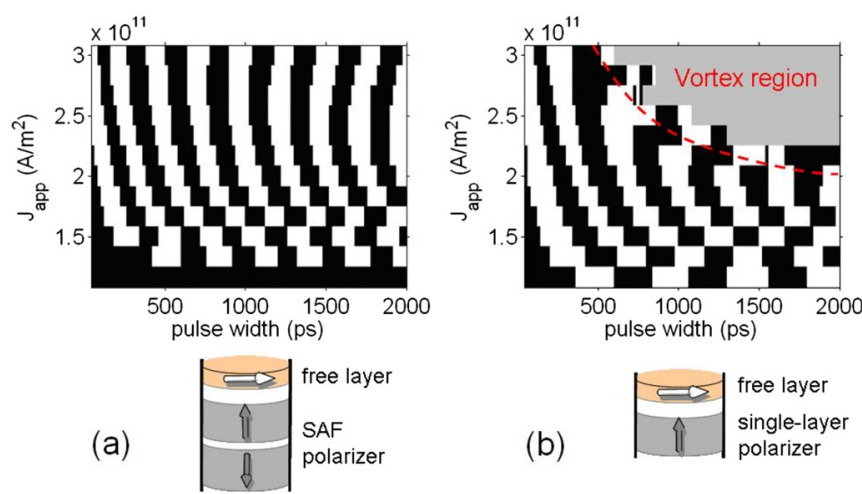

FIG. 2. (Color online) Switching probability as a function of pulse width and current density for a SAF-polarizer (a) and a single-layer polarizer (b). Black: $P_{s}=0$ (the final state is the same as the initial state). White: $P_{s}=1$ (the final state is opposite to the initial state). Gray: the final state is a vortex. The current density ranges from $1.17 \times 10^{11}-3 \times 10^{11} \mathrm{~A} / \mathrm{m}^{2}$ by 0.17 $\times 10^{11} \mathrm{~A} / \mathrm{m}^{2}$ step. The pulse width ranges from 50 to 1990 ps by $20 \mathrm{ps}$ step.

The mean value of the Oersted field is $1.5-4 \mathrm{kA} / \mathrm{m}$ in the range of current considered and was therefore neglected. The material parameters are saturation magnetization $\mathrm{M}_{\mathrm{s}}$ $=1040 \mathrm{kA} / \mathrm{m}$, exchange constant $A_{e x}=1.3 \times 10^{-11} \mathrm{~J} / \mathrm{m}$, magnetocrystalline anisotropy $K_{u}=0 \mathrm{~J} / \mathrm{m}^{3}$, and damping parameter $\alpha=0.02$. The FL magnetization dynamics obeys the Landau-Lifshitz-Gilbert-Slonczewski equation

$$
\begin{aligned}
\frac{\partial \mathbf{M}}{\partial t}= & -\gamma_{0}\left(\mathbf{M} \times \mathbf{H}_{\mathrm{eff}}\right)+\frac{\alpha}{\mathbf{M}_{\mathbf{S}}}\left(\mathbf{M} \times \frac{\partial \mathbf{M}}{\partial t}\right) \\
& +\gamma_{0} a_{J}[\mathbf{M} \times(\mathbf{M} \times \mathbf{P})],
\end{aligned}
$$

where $\gamma_{0}$ is the gyromagnetic factor and $\mathbf{H}_{\text {eff }}$ is the effective field. $\mathrm{a}_{\mathrm{J}}$ depends on the angle between the FL magnetization $\mathbf{M}$ and the spin polarization vector $\mathbf{P}$, as explained in Ref. 22.

Results of the micromagnetic simulations are plotted in Figs. 2 and 3. Due to the slight in-plane shape anisotropy, the critical current $\mathrm{I}_{\mathrm{c} 0}$ for the onset of the precessional motion of the FL magnetization is finite ${ }^{18}$ and, respectively, equal to $1.2 \times 10^{11} \mathrm{~A} / \mathrm{m}^{2}$ in case (i) [Fig. 2(a)] and 1.1 $\times 10^{11} \mathrm{~A} / \mathrm{m}^{2}$ in case (ii) [Fig. 2(b)]. In Fig. 2(a), when the current is increased, the precession frequency reaches a maximum and then starts decreasing slightly. This is explained by a change in the micromagnetic configuration of the FL. ${ }^{22}$ At moderate current density $\left(1.2-2.10^{11} \mathrm{~A} / \mathrm{cm}^{2}\right)$, the switching probability diagram is consistent with the inverse proportional relationship between switching current and pulse duration expected from macrospin behavior. ${ }^{18}$ For twice the critical current $\mathrm{I}_{\mathrm{c} 0}$, the magnetization switches in less than $100 \mathrm{ps}$, which is a very promising result for ultrafast MRAM or logic complementary metal-oxide semiconductor/magnetic devices implementing this precessional STT switching. Fig. 3(a) shows snapshots of the FL micromagnetic configuration at various stages of its precessional motion. Some magnetic distortions are observed but the overall behavior remains close to macrospin over the whole duration of the pulse. It is interesting to note that even after switching off the current, the FL magnetization keeps on precessing for at least one period since it takes time for the FL magnetization to return into the plane of the layer. In case (ii), at high current density $\left(>2 \mathrm{I}_{\mathrm{c} 0}\right)$, a vortex forms after a few $(\sim 4)$ precessional cycles for both positive [Fig. 3(b)] 

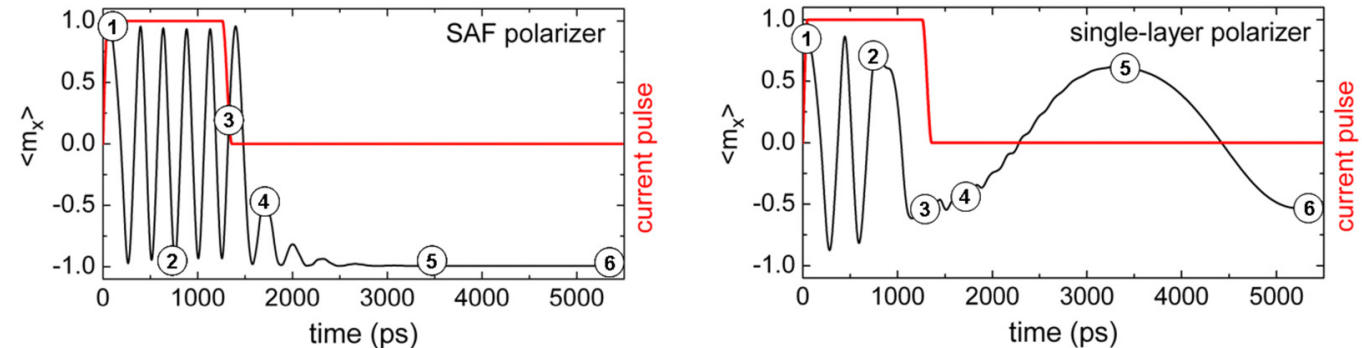

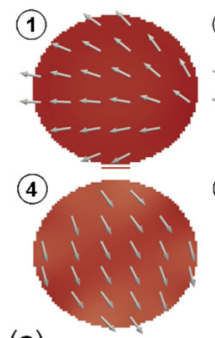

(a)

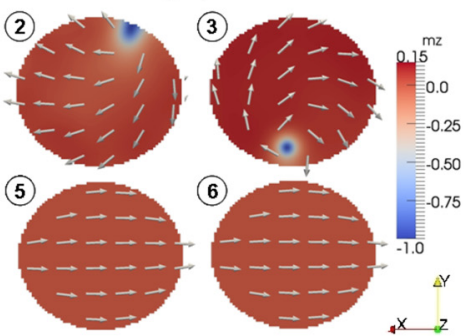

$\stackrel{2}{*} \geq$

(b)

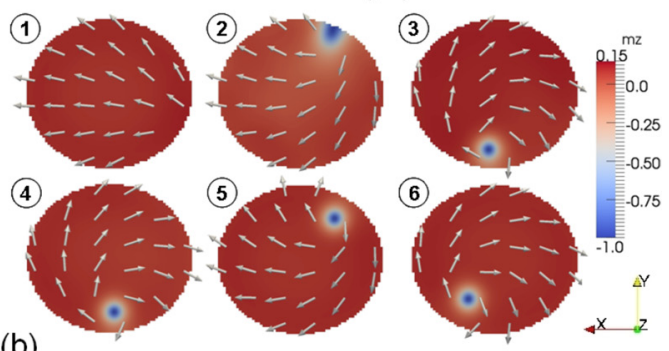

FIG. 3. (Color online) Simulations for a current density $\mathrm{J}_{\mathrm{app}}=2.5 \times 10^{11} \mathrm{~A} / \mathrm{m}^{2}$ and pulse length $\Delta \mathrm{t}=1210$ ps with a SAF polarizer (a) and a single-layer polarizer (b). The color scale represents the out-of-plane component of the magnetization. In (b), when the current is off, the vortex describes a spiral around the FL center. The system is at static equilibrium when the vortex reaches the center.

and negative (not shown) currents. It means that the coherence of the FL magnetization dynamics is strongly affected by the PL stray field. When the current is switched off, the vortex core keeps on rotating at much slower frequency on a trajectory of gradually decreasing radius. Its direction of rotation is determined by the surrounding in-plane spins which "drag" it. ${ }^{22}$ The vortex is then static when it reaches the center. Experiments show that the final state is one of the two in-plane equilibrium state and cannot be a vortex. In fact, the vortex state is an energy minimum, but not a global minimum. At room temperature, thermal fluctuations are expected to overcome the energy barrier, such that the system would end up in one of the two in-plane equilibrium states (global minima) with equal probability. Therefore, for I $>2 \mathrm{I}_{\mathrm{c} 0}$, the coherence is lost for long pulses, and the switching probability goes to 0.5 . This is in good agreement with the experimental observation of a much faster damping of the oscillation of probability when using a single layer perpendicular polarizer.

In conclusion, we have carried out experiments in $\mathrm{PL}_{\perp} / \mathrm{Cu} / \mathrm{FL} / \mathrm{Cu} / \mathrm{AL}$ spin valves, and we have shown that the coherence of the precessional motion of the FL magnetization is strongly enhanced when the perpendicular polarizer is a SAF. Micromagnetic simulations were performed demonstrating the effect of the stray field from the perpendicular polarizer on the FL. The coherence of the magnetization dynamics is affected by the dipolar field, and leads to a rapid damping of the oscillations in switching probability as a function of pulse duration. Therefore, the use of a SAF polarizer improves the switching reproducibility in these ultrafast switching MRAM cells. It is interesting to note that the improved coherence of the precession likely means a correlative narrower linewidth when using this type of structures as frequency tunable RF oscillators.

The authors thank Benjamin Seguret for his help with Perl scripts. This work was supported by the European Commission through the ERC-2009-AdG 246942 HYMAGINE.
${ }^{1}$ J. C. Slonczewski, J. Magn. Magn. Mater. 159, L1 (1996).

${ }^{2}$ L. Berger, Phys. Rev. B 54, 9353 (1996).

${ }^{3}$ D. Houssameddine, U. Ebels, B. Delaët, B. Rodmacq, I. Firastrau, F Ponthenier, M. Brunet, C. Thirion, J. P. Michel, L. Prejbeanu-Buda, M. C. Cyrille, O. Redon, and B. Dieny, Nature Mater. 6, 447 (2007).

${ }^{4}$ A. A. Tulapurkar, T. Devolder, K. Yagami, P. Crozat, C. Chappert, A. Fukushima, and Y. Suzuki, Appl. Phys. Lett. 85, 5358 (2004).

${ }^{5}$ T. Devolder, J. Hayakawa, K. Ito, H. Takahashi, S. Ikeda, P. Crozat, N. Zerounian, J. V. Kim, C. Chappert, and H. Ohno, Phys. Rev. Lett. 100, 057206 (2008).

${ }^{6}$ T. Devolder, C. Chappert, P. Crozat, A. Tulapurkar, Y. Suzuki, J. Miltat, and K. Yagami, Appl. Phys. Lett. 86, 062505 (2005).

${ }^{7}$ Y. T. Cui, J. C. Sankey, C. Wang, K. V. Thadani, Z. P. Li, R. A. Buhrman, and D. C. Ralph, Phys. Rev. B 77, 214440 (2008).

${ }^{8}$ T. Devolder, P. Crozat, J. V. Kim, C. Chappert, K. Ito, J. A. Katine, and M. J. Carey, Appl. Phys. Lett. 88, 152502 (2006).

${ }^{9}$ T. Devolder, C. Chappert, J. A. Katine, M. J. Carey, and K. Ito, Phys. Rev. B 75, 064402 (2007).

${ }^{10}$ Y. T. Cui, G. Finocchio, C. Wang, J. A. Katine, R. A. Buhrman, and D. C. Ralph, Phys. Rev. Lett. 104, 097201 (2010).

${ }^{11}$ T. Devolder, C. Chappert, and K. Ito, Phys. Rev. B 75, 224430 (2007).

${ }^{12}$ I. N. Krivorotov, N. C. Emley, J. C. Sankey, S. I. Kiselev, D. C. Ralph, and R. A. Buhrman, Science 307, 228 (2005).

${ }^{13}$ G. Finocchio, I. N. Krivorotov, L. Torres, R. A. Buhrman, D. C. Ralph, and B. Azzerboni, Phys. Rev. B 76, 174408 (2007).

${ }^{14}$ S. Garzon, L. Ye, R. A. Webb, T. M. Crawford, M. Covington, Phys. Rev. B 78, 180401(R) (2008).

${ }^{15}$ J. C. Slonczewski, U.S. Patent No. 5,695,864 (9 December 1997).

${ }^{16}$ O. Redon, B. Dieny, and B. Rodmacq, U.S. Patent No. 6,532,164 B2 (11 March 2003).

${ }^{17}$ A. D. Kent, B. Özyilmaz, and E. Del Barco, Appl. Phys. Lett. 84, 3897 (2004).

${ }^{18}$ K. J. Lee, O. Redon, and B. Dieny, Appl. Phys. Lett. 86, 022505 (2005).

${ }^{19}$ D. E. Nikonov, G. I. Bourianoff, G. Rowlands, and I. N. Krivorotov, J. Appl. Phys. 107, 113910 (2010).

${ }^{20}$ O. J. Lee, V. S. Pribiag, P. M. Braganca, P. G. Gowtham, D. C. Ralph, and R. A. Buhrman, Appl. Phys. Lett. 95, 012506 (2009).

${ }^{21}$ C. Papusoi, B. Delaët, B. Rodmacq, D. Houssameddine, J. P. Michel, U. Ebels, R. C. Sousa, L. Buda-Prejbeanu, and B. Dieny, Appl. Phys. Lett. 95, 072506 (2009).

${ }^{22}$ I. Firastrau, D. Gusakova, D. Houssameddine, U. Ebels, M.-C. Cyrille, B. Delaet, B. Dieny, O. Redon, J.-Ch. Toussaint, and L. D. Buda-Prejbeanu, Phys. Rev. B 78, 024437 (2008). 